\title{
Screening of Painful Hip Joint with Radiography and MRI
}

\author{
Shriyash Bapurao Pinglikar ${ }^{1}$ Nagesh Dinesh Seth², Priscilla Chandrashekhar Joshi³, John D’Souza ${ }^{4}$ \\ ${ }^{1}$ Department of Radiology, Bharati Vidyapeeth Deemed to be University, Pune, Maharashtra, India. \\ 2Department of Radiology, Bharati Vidyapeeth Deemed to be University, Pune, Maharashtra, India. \\ ${ }^{3}$ Department of Radiology, Bharati Vidyapeeth Deemed to be University, Pune, Maharashtra, India. \\ ${ }^{4}$ Department of Radiology, Bharati Vidyapeeth Deemed to be University, Pune, Maharashtra, India.
}

\section{ABSTRACT}

\section{BACKGROUND}

The basic tool for hip joint study is radiography. Pelvic radiograph helps in deciding whether the hip joint is normal and also includes opposite hip joint for comparison. It also helps in identification of signs of degenerative and inflammatory changes, avascular necrosis, soft tissue calcification and bone tumours. The investigation of choice to assess the bone marrow, acetabular labrum, articular cartilage, periarticular muscles, tendons, ligaments and bursae is MRI. Pain at hip joint has different aetiologies divided into intra-articular, peri-articular and extra-articular conditions in adults and children. This study is being done to evaluate painful hip joint with radiography and MRI.

\section{METHODS}

50 cases of painful hip joint referred to the Radiology Department, Bharati Hospital were evaluated with radiography and MRI over a period of 24 months.

\section{RESULTS}

Out of 50 patients 20 were diagnosed as AVN, 10 cases showed changes of osteoarthritis, 7 cases had joint effusion, 5 cases were of fracture of neck of femur, 4 cases had sacroiliitis and 4 cases revealed metastatic disease of hip joint. Of the 50 cases that presented with pain in hip joint, 26 cases showed radiographic findings. The rest 24 radiographs were normal.

\section{CONCLUSIONS}

Radiography is easily available and economical for imaging the hip joint. Radiography is the initial investigation for evaluation of joint pain. MRI is noninvasive. There is no radiation exposure. It is a valuable tool in diagnosing hip pain when radiography shows subtle or no findings. MR imaging is the most sensitive imaging modality for bone pain.

\section{KEY WORDS}

Hip Joint, Pain, Radiography, X-Ray, MRI
Corresponding Author: Nagesh Dinesh Seth, Department of Radiology, Bharati Vidyapeeth Deemed to be University, Pune, Maharashtra, India. E-mail: nvseth@gmail.com

DOI: $10.14260 /$ jemds/2020/279

Financial or Other Competing Interests: None.

How to Cite This Article: Pinglikar SB, Seth ND, Joshi PC, et al. Screening of painful hip joint with radiography and MRI. J. Evolution Med. Dent. Sci. 2020;9(15):1286-1290, DOI: 10.14260/jemds/2020/279

Submission 28-01-2020, Peer Review 21-03-2020, Acceptance 27-03-2020, Published 13-04-2020.

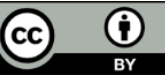




\section{BACKGROUND}

The hip joint is large, bears weight and its shape with surrounding strong ligaments and muscles provides stability. It is a rigid ball-and-socket type of synovial joint. The acetabular cartilage is horse-shoe-shaped with a central deficient part that is without cartilage coverage that does not articulate with the femoral head (fossa acetabuli). The femoral head is completely covered with hyaline cartilage except for the insertion of the ligamentum teres. The hip joint articular cartilage is thin in comparison to other joints with its maximum thickness ventro-cranially in the acetabulum and ventro-laterally on the femoral head. ${ }^{1}$ The basic tool for hip joint study is radiography. ${ }^{2} \mathrm{~A}$ number of $\mathrm{X}$-ray projections have been devised to image a painful hip joint. The standard $\mathrm{X}$-ray view is anteroposterior view. ${ }^{3}$ It helps in assessment of joint space and acetabular integrity.

The investigation of choice to assess the bone marrow, acetabular labrum, articular cartilage, peri-articular muscles, tendons, ligaments and bursae is magnetic resonance imaging (MRI). ${ }^{4}$ MRI hip was one of the earliest modalities used in the diagnosis of musculoskeletal pathologies. In the diagnosis of musculoskeletal pathologies, hip imaging with MRI was the earliest. The hip pain management in case of nonspecific history, unconvincing clinical findings and normal radiograph is difficult. In various aetiologies like trauma, arthritis, avascular necrosis, metastasis the radiographic findings are very subtle. 5

Pain at hip joint has different aetiologies divided into intra-articular, peri-articular and extra-articular conditions in adults and children. In adults the intra-articular conditions are arthritis, labral tears, avascular necrosis and loose bodies. Peri-articular conditions that may cause pain at hip joint are tendinitis and bursitis. Extra-articular conditions are referred pain from lumbar spine, Sacro-iliac joint and nerve entrapment syndrome. ${ }^{6}$ MRI provides good soft tissue contrast and resolution which helps in diagnosing soft tissue abnormalities like bursitis and muscle tears. ${ }^{7}$ MRI provides information regarding osteoarthritis, stress fractures, avascular necrosis affecting the articulating bones. ${ }^{8}$

Thus, this study was done to evaluate painful hip joint with radiography and MRI. Our objectives were to establish the agreement between findings of radiograph and MRI in evaluation of painful hip joint and to estimate the role of MRI in early evaluation of painful hip joints and to give differential diagnoses.

\section{METHODS}

This was an observational cross-sectional study carried out over a period of 2 years from September 2017 to august 2019. The study was initiated after obtaining institutional ethics committee approval from Bharati Vidyapeeth Deemed University Medical College and Hospital. Written informed consent was obtained from all the 50 patients involved in the study.

\section{Inclusion Criteria}

Patients more than 18 years, either sex and any profession had undergone radiography and MRI for pain at hip joint.

\section{Exclusion Criteria}

Intra ocular implant, Aneurysm clips, cardiac pacemakers

\section{Technique and Instrumentation}

$\mathrm{X}$ - ray pelvis with both hips AP view obtained on Konica Minolta DR system. MR Imaging was done with 1.5 Tesla Philips Achieva Machine imaging system using surface coils and spine coils. The following sequences were selected as required. Contrast sequences were acquired as and when required.

\begin{tabular}{|cccc|}
\hline Sequences & TR & TE & Slice Thickness \\
T1W Axial & $500-700 \mathrm{~ms}$ & $18 \mathrm{~ms}$ & $1-3 \mathrm{~mm}$ \\
T1 Coronal & $500-700 \mathrm{~ms}$ & $18 \mathrm{~ms}$ & $1-3 \mathrm{~mm}$ \\
T2W axial & $1000-1500 \mathrm{~ms}$ & $100 \mathrm{~ms}$ & $1-3 \mathrm{~mm}$ \\
T2W Coronal & $1000-1500 \mathrm{~ms}$ & $100 \mathrm{~ms}$ & $1-3 \mathrm{~mm}$ \\
STIR Coronal & $2700-6000 \mathrm{~ms}$ & $30 \mathrm{~ms}$ & $3-5 \mathrm{~mm}$ \\
PD Sagittal & $2300-6500 \mathrm{~ms}$ & $30 \mathrm{~ms}$ & $3-5 \mathrm{~mm}$ \\
FFE Axial & $500 \mathrm{~ms}$ & $9-10 \mathrm{~ms}$ & $1-3 \mathrm{~mm}$ \\
\hline \multicolumn{4}{c}{ Sequences } \\
\hline
\end{tabular}

\section{Statistical Analysis}

Descriptive statistics were used to describe the data. Frequency with percentage for qualitative data and mean with standard deviation for quantitative data were used. As we screened the patients with painful hip with radiography and MRI, only sensitivity analysis was done to see agreement between radiography and MRI.

\section{RESULTS}

Total 50 cases presented with pain in hip joint underwent both radiography and MRI. Maximum (40\%) patients were in $3^{\text {rd }}$ and $4^{\text {th }}$ decade of life followed by $36 \%$ patients in 18 - 30 years of age group. There was male preponderance in the study._20 cases had AVN, 10 cases showed changes Osteoarthritis, 7 cases were of Joint effusion, 5 cases had fracture neck of femur, 4 cases diagnosed as sacroiliitis and remaining 4 cases revealed metastatic disease to hip joint. Out of 20 cases diagnosed as AVN, only 30\% cases had findings on radiography, whereas all were diagnosed on MRI, confirming that MRI is more sensitive for the detection and also assessing in staging of AVN.

Although all the 10 cases of osteoarthritis were detected both on radiography and MRI, MRI revealed better delineation of cartilage destruction, accurate pathological involvement and staging of osteoarthritis. Out of 7 cases who showed joint effusion, only $28.5 \%$ cases were diagnosed on radiography showing widened tear drop distance, whereas $100 \%$ were diagnosed on MRI proving its higher sensitivity. Fracture of neck of femur was diagnosed in 5 cases of which radiography detected $80 \%$ and MRI detected all, thereby making it superior to radiography in evaluation of fracture neck of femur.

Out of all the 4 cases of sacroiliitis, none of the case was detected on radiography. All the 4 cases were detected MRI. Role of MRI in the evaluation of sacroiliitis is more preferred. 4 other cases showed metastasis to the hip joint and pelvic bones. Radiography delineated well osteolytic and osteoblastic lesions. MRI helps in the evaluation of the involvement articular cartilage in the form of $\mathrm{T} 2 \mathrm{~W}$ 
hyperintensity. It also helps in evaluation of soft tissue involvement along with detection of bone marrow oedema. To establish an agreement between the finding of radiography and MRI, out of 50 cases that were presented with pain in hip joint 26 cases showed radiographic findings. The rest of the 24 radiographs appeared normal. All the 50 cases were detected on MRI. Sensitivity of radiography in detecting overall hip joint pathologies was only $52 \%$. So, our study proves that MRI is highly sensitive in the evaluation of causes painful hip joint.
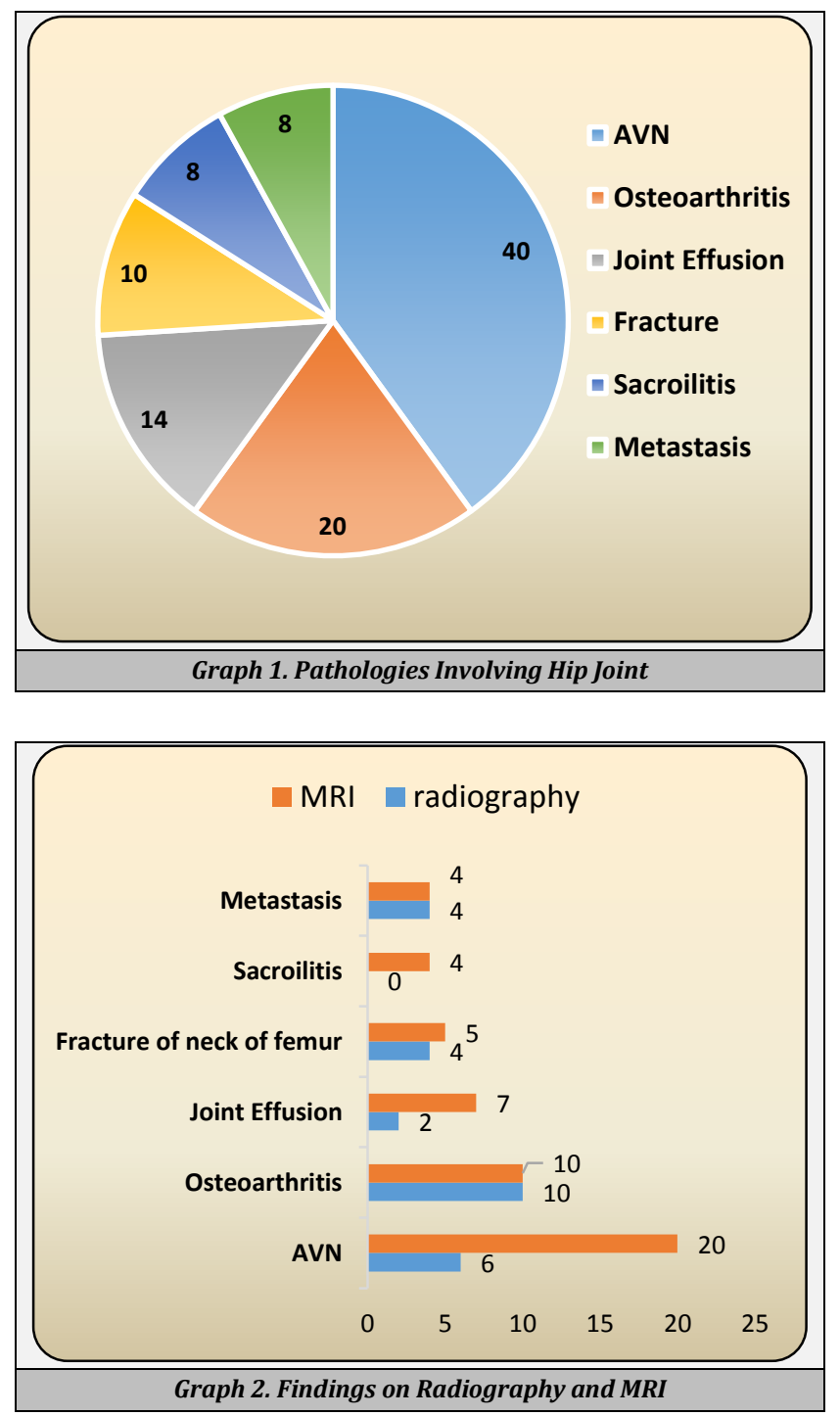

\section{DISCUSSION}

Our study group comprised 50 patients of age more than 18 years. The mean age was 38.2 years $(S D \pm 16.99$ ) with three groups mainly 18 to 30 years, $31-50$ years and $>50$ years, most patients were in the age group $31-50$ years. Out of this 35 were males and 15 were females. These similar findings were also observed in study done by Tushar Kalekar et al. ${ }^{9}$

Sensitivity of radiography in detecting overall hip joint pathologies was only $52 \%$. Our study thus proves that MRI is highly sensitive in the evaluation of causes painful hip joint. Previous studies done by Tushar Kalekar et al and Drar HA et al10 in 2018 and in 2014 also proved that MRI is the most sensitive in diagnosing cases of hip pain.

\section{Avascular Necrosis of Femoral Head}

In our study we found that AVN of femoral head is the most common pathology for painful hip joint. Out of 20 cases of AVN only 6 cases were diagnosed on radiography whereas MRI diagnosed all, Bassett, L et all ${ }^{11}$ in their study also concluded that MRI is most sensitive in diagnosing AVN. MRI evaluates better in the staging of AVN than X-Ray that helps in appropriate treatment plan by the clinician based on the stage of AVN. Zoia Stoica et al ${ }^{12}$ in 2009 also stated same findings. Robinson $\mathrm{HJ}$ Jr. et al, 13 in their second phase studied 23 suspected cases of early-stage of avascular necrosis of the femoral head. They found that radiography showed no or subtle changes. These patients were then repeatedly studied with interval radiographs and MRI. Out of the 23 hips, 18 (78 $\%$ ) had findings of avascular necrosis of femoral head on magnetic resonance imaging. This is close to our study where all the 20 cases were detected on MRIs (100\%), in comparison to radiography which detected only 6 cases with sensitivity of 30\%. Resnik D. et al ${ }^{14}$ in 1995 also found sensitivity of radiography to be $41 \%$ which is nearly same as in our study.

\section{Osteoarthritis}

Our study detected 10 cases of osteoarthritis. Both radiography and MRI showed findings of osteoarthritis in all the 10 cases, however they differed in staging of osteoarthritic changes. MRI is better in delineation of cartilage destruction and thus leads to better staging of osteoarthritis. This was also proved by Drar HA et al ${ }^{10}$ in 2014.

\section{Joint Effusion}

MRI is more sensitive in comparison to radiography in evaluation of joint effusion. It also helps in grading the joint effusion. This was also stated in study done by Tushar Kalekar et $\mathrm{al}^{9}$ in 2018. Our study concurred with the Sweeney JP et al study. ${ }^{15}$ In their study they found that widened tear drop distance is an indicator of joint effusion which was confirmed in our study. However, radiography detected only $28.5 \%$ of the joint effusions using this criterion.

\section{Fracture}

Deutsch et al ${ }^{16}$ in their study showed that fractures that are difficult to appreciate on CT such as, stress fractures, nondisplaced fractures and subtle fractures in severely osteopenic patients, are easily recognized at MR imaging because of the marrow changes. Magnetic resonance imaging facilitates the early diagnosis of stress fracture as it results in bone marrow oedema which is best depicted on T1WI, fat suppressed T2WI and STIR images. Out of 50 cases 5 cases had fracture of neck of femur. Out of all the 5 cases detected on MRI, only 4 cases were detected on radiography. Drar HA et $\mathrm{al}^{10}$ in their study stated that MRI has $100 \%$ sensitivity in detection of fractures. In our study also MRI detected all the cases of fractures. 


\section{Sacroiliitis}

4 cases showed imaging findings of sacroiliitis in the study. None of the case was detected on radiography whereas MRI detected all. The likely reason for this would be patient visiting to us in an early stage of the disease. MRI showed bone marrow oedema on STIR images and bone marrow contrast enhancement on a T1-weighted sequence. SudołSzopińska I et al ${ }^{17}$ studied 101 suspected cases of sacroiliitis. In their study out of 86 patients displaying no sacroiliitis on radiograms, MRI showed sacroiliitis in 34 patients (39.5\%). This contradicts to our study findings, where MRI detected all the cases. The reason may be smaller sample size of our study.

\section{Metastasis}

Out of 50 cases, 4 cases showed metastatic disease. All The cases were detected on radiography and MRI. Two cases showed osteoblastic metastasis and 2 cases show osteolytic metastasis. MRI is better in the evaluation of metastatic lesions as it helps in detection of soft tissue involvement, which is difficult to detect on radiograph, can aid in the appropriate treatment plan. Vaghamashi A et al ${ }^{18}$ showed MRI is more sensitive in the detection of bone metastases because earlier marrow abnormalities may be identified and show both lytic and sclerotic lesions. In their study 1 case had metastatic lesion out of 52 total patients. In our study 4 cases, out of 50 were having metastatic lesions.

\section{CONCLUSIONS}

Radiography is an easily available and economical investigation for imaging a painful hip joint. Radiography is amongst the initial investigations done for evaluation of pain of any cause. MRI is a non-invasive and valuable tool in evaluating hip pain when radiography shows very subtle or no findings. There is no radiation exposure. MR imaging is the most sensitive imaging modality for diagnosing AVN. MRI is better in detection of osteoarthritis, delineation of cartilage destruction and reveals accurate pathological involvement and staging of osteoarthritis which helps in appropriate plan of treatment or intervention by the clinician. MRI is more sensitive in detection of joint effusion in comparison to radiography. It also helps grading of joint effusion. MRI is better in the detection of fracture of neck of femur like occult fracture and stress fractures. In comparison to radiography, MRI is better in the evaluation of sacroiliitis in early stages. MRI is superior in the evaluation of metastatic lesions by not only detecting the abnormal signal intensity lesions, also evaluates the cartilage and the extent of soft tissue involvement accurately, which helps in formulating an appropriate treatment plan.

Small study population with only adults and symptomatic patients being included [asymptomatic controls were not included in the study] are the limitations of this study.

\section{REFERENCES}

[1] Zilkens C, Miese F, Jäger $M$, et al. Magnetic resonance imaging of hip joint cartilage and labrum. Orthopedic Reviews (Pavia) 2011;3(2):e9.

[2] Karrasch C, Lynch S. Practical approach to hip pain. Medical Clinics of North America 2014;98(4):73754.

[3] Clohisy JC, Carlisle JC, Beaulé PE, et al. A systematic approach to the plain radiographic evaluation of the young adult hip. The Journal of Bone and Joint Surgery Am 2008;90(Suppl 4):47-66.

[4] Chiamil SM, Abarca CA. Imaging of the hip: a systematic approach to the young adult hip. Muscles, Ligaments And Tendons Journal 2016;6(3):265-80.

[5] Manaster BJ. Adult chronic hip pain: radiographic evaluation. From the RSNA Refresher Courses. Radiological Society of North America. Radiographics 2000;20 Spec No.:S3-S25.

[6] Ghebontni L, Roger B, El-Khoury J, et al. MR arthrography of the hip: normal intra-articular structures and common disorders. European Radiology 2000;10(1):83-8.

[7] Boutry N, Paul C, Leroy X, et al. Rapidly destructive osteoarthritis of the hip: MR imaging findings. AJR Am J Roentgenol 2002;179(3):657-63.

[8] Verbeeten KM, Hermann KL, Hasselqvist M, et al. The advantages of MRI in the detection of occult hip fractures. Eur Radiol 2005;15(1):165-9.

[9] Kalekar T, Shriramwar P. MRI evaluation of nontraumatic painful hip joint. International Journal of Contemporary Medicine Surgery and Radiology 2018;3(1):155-60.

[10] Drar HA, Mohammed BA, Ali ZA. The role of MRI in the evaluation of painful hip joint (MRI of hip joint). International Journal of Medical Imaging 2014;2(3):7782.

[11] Bassett LW, Gold RH, Reicher M, et al. MRI in the early diagnosis of ischemic necrosis of the femoral head. Preliminary results. Clin Orthop Relat Res 1987;(214):237-48.

[12] Stoica Z, Dumitrescu D, Popescu M, et al. Imaging of avascular necrosis of femoral head: familiar methods and newer trends. Current Health Sciences Journal 2009;35(1):23-8.

[13] Robinson HJ Jr, Hartleben PD, Lund G, et al. Evaluation of magnetic resonance imaging in the diagnosis of osteonecrosis of the femoral head. Accuracy compared with radiographs, core biopsy and intraosseous pressure measurements. J Bone Joint Surg Am 1989;71(5):650-63.

[14] Resnick D, Niwayama G. Osteonecrosis: diagnostic techniques, special situations and complications. In: Resnick D, edr. Diagnosis of bone and joint disorders. Philadelphia: WB Saunders Co, 1995: p. 3495.

[15] Sweeney JP, Helms CA, Minagi H, et al. The widened teardrop distance: a plain film indicator of hip joint effusion in adults. American Journal of Roentgenology 1987;149(1):117-9.

[16] Deutsch AL, Mink JH, Waxman AD. Occult fractures of the proximal femur: MR imaging. Radiology 1989;170(1 Pt 1):113-6. 
[17] Sudoł-Szopińska I, Kwiatkowska B, WłodkowskaKorytkowska M, et al. Diagnostics of sacroiliitis according to ASAS criteria: a comparative evaluation of conventional radiographs and MRI in patients with a clinical suspicion of spondyloarthropathy. preliminary results. Polish Journal of Radiology 2015;80:266-76.

[18] Vaghamashi A, Bhatt J, Doshi J, et al. MRI in Evaluation of painful hip Joint. IOSR-JDMS 2017;16(5):85-96. 